\title{
The hominid thumb and its influence on the employment during evolution
}

\author{
J.H. Villafañe ${ }^{1}$, R. Cantero-Tellez ${ }^{2}$, P. Berjano ${ }^{3}$ \\ ${ }^{1} / R C C S$ Fondazione Don Carlo Gnocchi, Milan, Italy; ${ }^{2}$ Physical Therapist University of Malaga, Spain; \\ IIRCCS Istituto Ortopedico Galeazzi, Milan, Italy
}

\section{SHORT REPORT}

T he ability of modern humans to manipulate, modify, and utilize their physical environment is unparalleled amongst primates. The fish Rhipidistia, our earliest known ancestor, had pectoral fins that phylogenetically preceded the arm, which then developed from the high cervical segments. Fish have no neck and the bones of their pectoral fins are articulated with the skull. Opposed to this, the intrinsic muscles of the hand in the human are innervated by the two lower branches of the brachial plexus (1). Hands perform a vital role in most daily human activities (2).

The hominids living at the end of the Miocene about 15 million years ago began to develop bipedal locomotion, liberating their hands for independent use. From an evolutionary stand point, the finger bones straightened the curvature that was used to grasp tree branches, and the thumb, formed last, receded in length, and diverged from the adjacent digits (3).

This could be the first succession of musculoskeletal adaptations for improved function and survival to have been described in the course of their evolution. The evolution of musculoskeletal adaptations into activity-related wear of the hand joints can be demonstrated nowadays with activityrelated hand pathologies. Furthermore, in this age of handheld electronic gadgets, for many individuals the thumb is repeatedly used to text on smartphones countless times per day. Adults using mobile phones punch out numbers with their thumbs and develop synovitis in their carpometacarpal joints. The influence of activity-related wear on the development of pathologies might have already been present in the early stages of the evolution of the hand, such as those that occurred to primates when they began to develop bipedal locomotion, liberating their hands for independent use (4).

That the evolution of the human hand has given our species the opportunity to evolve into more complex activities and societies is a fact, and this could have influenced the development of our species' brain. But, on an individual scale, does the hand shape our brain activity? Is there a correlation between hand wear and brain activity as shown by brain mapping? We already know that orthopaedic disorders of the hand that reduce the patients' motor repertoire induce neurofunctional changes in the cortical representation of hand movements. Thus, hand wear influences cortical motor mapping (5). Widespread hyperalgesia is a feature of OA. OA is a chronic disease in which mechanisms of pain are not fully understood (6). Association between clinical and neurophysiological outcomes is highly important to improve therapeutic approaches for hand OA. Villafañe et al., reported that radial nerve mobilisation on the affected side induced bilateral hypoalgesic effects in people with carpometacarpal osteoarthritis (7). We already know that, although the perceptual implications of the anatomical changes and the rules that facilitate transfer of learning to other tactile abilities remain unclear, repetitive tactile stimulation on the hand modifies neural representations in primary somatosensory cortex (SI) of non-human primates (8). It is likely that the development of new tech-
Corresponding author: Jorge H. Villafañe

IRCCS Fondazione Don Carlo Gnocchi, Milan, Italy

E-mail: mail@villafane.it 
nology brings an opportunity to reduce hand wear. Dictation software can make it unnecessary to use pencils, keyboards or tactile screens of smartphones, robots will clean our pavements without any involvement of our hands, self-driving cars will free our hands from steering wheels, iris or face recognition will make unnecessary the use of fingerprints (though it is arguable whether this is a significant source of wear), surgical robots will amplify our hand strength and preserve surgeons from trapeziometacarpal arthritis. One question still remains open: just as hand wear can induce brain plasticity into less functional neuronal circuits, will hand underuse carry as a consequence an underdevelopment of our brain? In other words, by freeing us from hand use, is technology going to reduce our brain functions? Only time and science will answer this question.

Contributions: JHV, design of the study and interpretation of the results; write, submit and tracking of manuscript; RC, interpretation of the results; write, submit and tracking a manuscripts; $\mathrm{PB}$, write, submit and tracking of manuscript.

Conflict of interest: the authors declare no conflict of interest.

\section{REFERENCES}

1. Diogo R, Wood B. Soft-tissue anatomy of the primates: phylogenetic analyses based on the muscles of the head, neck, pectoral region and upper limb, with notes on the evolution of these muscles. J Anat. 2011; 219: 273-359.

2. Marzke MW, Toth N, Schick K, et al. EMG study of hand muscle recruitment during hard hammer percussion manufacture of Oldowan tools. Am J Phys Anthropol. 1998; 105: 315-32.

3. Ladd AL. Editorial comment: Symposium: Thumb carpometacarpal arthritis. Clin Orthop Relat Res. 2014; 472: 1093-4.

4. Bertozzi L, Valdes K, Vanti C, et al. Investigation of the effect of conservative interventions in thumb carpometacarpal osteoarthritis: systematic review and meta-analysis. Disabil Rehabil. 2015; 37: 2025-43.

5. Gandola M, Bruno M, Zapparoli L, et al. Functional brain effects of hand disuse in patients with trapeziometacarpal joint osteoarthritis: executed and imagined movements. Exp Brain Res. 2017; 235: 3227-41.

6. Villafane JH. Does "time heal all wounds" still have a future in osteoarthritis? Clin Exp Rheumatol. 2018; 36: 513.

7. Villafane JH, Bishop MD, Fernandez-de-LasPenas C, Langford D. Radial nerve mobilisation had bilateral sensory effects in people with thumb carpometacarpal osteoarthritis: a randomised trial. J Physiother. 2013; 59: 25-30.

8. Trzcinski NK, Gomez-Ramirez M, Hsiao SS. Functional consequences of experience-dependent plasticity on tactile perception following perceptual learning. Eur J Neurosci. 2016; 44: 2375-86. 